\title{
Screening for genes that are differentially- expressed between gastric cancer cells and gastric tumor sphere cells using the gene chip technique
}

\author{
W.H. Shi, Y.B. Yu, Z.-Y. Li, X.-C. Guo, Z.-L. Wei and Z.-H. Zheng \\ Department of Laboratory Animals, China Medical University, \\ Shenyang, China \\ Corresponding author: Wei-Hong Shi \\ E-mail: shiwhvip2@163.com \\ Genet. Mol. Res. 14 (4): 14893-14899 (2015) \\ Received June 9, 2015 \\ Accepted September 24, 2015 \\ Published November 18, 2015 \\ DOI http://dx.doi.org/10.4238/2015.November.18.54
}

\begin{abstract}
The purpose of this study was to screen for genes that were differentially expressed between a human gastric carcinoma cell line (HGC-27) and their tumor spheres, using the gene chip technique. The HGC-27 cells and tumor sphere cells were cultured in vitro in a sterile environment. Total RNA was extracted from both samples and purified using a standard TRIzol reagent. Total RNA was then hybridized onto a GeneChip, according to the standard protocols provided by the manufacturers of the GeneChip IVT Express Kit. The resulting fluorescence signals were analyzed and displayed using the Cluster and Treeview software programs. Under the criteria for significant differential expression ( $\geq 2$-fold difference), 610 up- and 1135 down-regulated genes were identified in tumor sphere cells, compared to HCG-27 cells. These genes were involved in cell growth, signal transduction, tumorigenesis, and many other functional aspects of tumor cells. In conclusion, a number of genes were differentially expressed in tumor sphere cells compared to
\end{abstract}


HCG-27 cells. In addition, we identified a close correlation between tumor sphere cells and tumorigenesis.

Key words: Gastric cancer cells; Tumor spheres; DNA microarray; Gene expression

\section{INTRODUCTION}

Gastric carcinoma is one of the most common cancers; the mortality rate of gastric carcinoma remains high despite the widespread availability of a number of conventional therapeutic strategies, including surgical ablation, chemotherapy, radiotherapy, immunotherapy, the use of traditional Chinese herbs, and gene therapy. However, the use of tumor stem cells (TSCs) (Reya et al., 2001) may provide a new approach for the pathogenesis, diagnosis, and treatment of gastric cancer.

Recent studies have revealed that tumor stem cells form spherical cell clusters (called tumor spheres) in suspension (Mimeault et al., 2007) when cultured in serum-free medium and when induced by growth factors, such as epidermal growth factor (EGF). Recent studies have also revealed that tumor spheres are enriched for cancer stem cells (Eramo et al., 2008; Yu et al., 2008; Bertolini et al., 2009; Levina et al., 2010).

In this study, the gene chip technique was used to screen for genes that were differentially expressed among human gastric carcinoma cells and their tumor sphere cells. We also attempted to identify the expression of specific genes in tumor sphere cells that were closely related to the occurrence and recurrence of gastric cancer, thereby providing a new approach for anti-cancer therapy.

\section{MATERIAL AND METHODS}

\section{Materials}

The human gastric carcinoma cell line HCG-27 was obtained from the cell bank of the Chinese Academy of Sciences. Fetal bovine serum (FBS; standard) and trypsin were purchased from Gibco (IL, USA). Dulbecco's modified Eagle's medium (DMEM), DMEM/F12, epidermal growth factor (EGF), and basic fibroblast growth factor (bFGF) were purchased from Invitrogen, while the GeneChip IVT Express Kit, GeneChip Hybridization, Wash and Stain Kit, GeneChip Hybridization Oven 640, GeneChip Fluidics Station 450, and GeneChip Scanner 3000 were obtained from Affymetrix.

\section{HCG-27 culture in serum-supplemented medium (SSM)}

HCG-27 cells were cultured in DMEM supplemented with $10 \%$ fetal bovine serum, $100 \mathrm{U} /$ $\mathrm{mL}$ penicillin and $100 \mu \mathrm{g} / \mathrm{mL}$ streptomycin (SSM) in a humidified $5 \% \mathrm{CO}_{2}$ incubator at $37^{\circ} \mathrm{C}$. The medium was changed according to the rate of cell growth, and/or based on the changes in the color of the medium. The cells were sub-cultured by trypsinization using $0.25 \%$ trypsin. HCG-27 cells in the log phase of growth were used in all experiments.

\section{HCG-37 culture in serum-free medium (SFM)}

HCG-27 cells were cultured in DMEM/F12 supplemented with $100 \mathrm{U} / \mathrm{mL}$ penicillin, $100 \mu \mathrm{g} /$ 
$\mathrm{mL}$ streptomycin, $20 \mathrm{ng} / \mathrm{mL}$ EGF, and $10 \mathrm{ng} / \mathrm{mL}$ bFGF, without serum. These cells were seeded on low cell-binding 6 -well plates $\left(10^{4}\right.$ cells $/ 2 \mathrm{~mL}$ in each well) and maintained in a humidified incubator $\left(5 \% \mathrm{CO}_{2}\right.$ at $\left.37^{\circ} \mathrm{C}\right)$. Fresh medium $(200 \mu \mathrm{L})$ was added to the culture each day, and the culture was analyzed every day for tumor sphere formation.

\section{Total RNA extraction}

Total RNA was isolated and further purified from HCG-27 cells and gastric tumor sphere cells using TRIzol reagent. The RNA purity was assessed by spectrometry, and the quality and integrity of total RNA was evaluated by formaldehyde denaturing agarose gel electrophoresis.

\section{Microarray hybridization of gene expression spectrum}

Complementary DNA (cDNA) was synthesized from the HCG-27 and tumor sphere samples using the GeneChip IVT Express Kit, according to the standard protocols detailed by the manufacturer. The cDNA was then labeled and hybridized to a zebrafish microarray for $18 \mathrm{~h}$ at $45^{\circ} \mathrm{C}$.

\section{Image scanning and data analysis}

The gene chips were washed and stained, and subsequently scanned using the GeneChip Scanner 3000. Images were generated, and the corresponding data was extracted. The differences in gene transcription levels among the samples were analyzed according to the corrected data.

\section{RESULTS}

\section{Tumor sphere formation}

HCG-27 cells grew as a monolayer attached to the surface of the culture container when cultured in serum-free medium. Compact tumor spheres were formed in the medium after about one week. Half the medium was removed and replaced with fresh medium in order to control the formation of tumor spheres; we observed a consistent increase in the tumor sphere size (Figure 1).

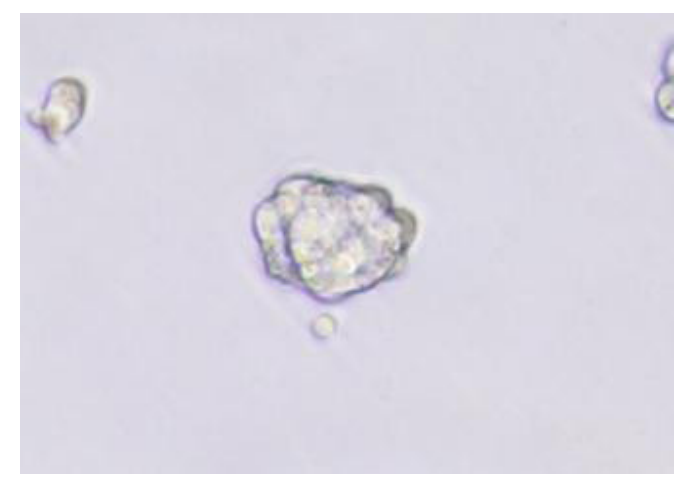

Figure 1. Tumor sphere of HGC-27 cell. 


\section{Total RNA extraction}

Total RNA extracted from HCG-27 cells and tumor sphere samples was analyzed using an ultraviolet spectrophotometer. We obtained an absorbance at $260 \mathrm{~nm} /$ absorbance at 280 $\mathrm{nm}$ (A260/A280) ratio of 1.812 for both samples, which validated the purity of the RNA (free of protein contamination). The quality and integrity of the total RNA was also assessed using formaldehyde agarose gel electrophoresis. The electrophoretogram of both samples showed distinctive bands corresponding to $28 \mathrm{~S}$ and $18 \mathrm{~S}$ ribosomal RNA; the ratio of intensities of these two bands was approximately 2:1. The result indicated that the total RNA extracted from both samples was intact and non-degraded, which was adequate for the subsequent gene chip experiment (Figure 2).

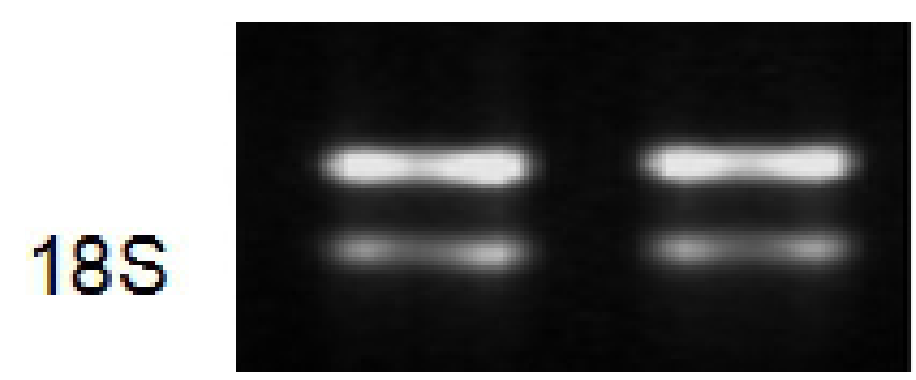

Figure 2. RNA electrophoretogram of HGC-27 cell and tumer sphere cell.

\section{Hybridization to gene chip}

The labeled cDNA was hybridized to its complement on the gene chip; the resulting signal intensities of the spots on the microarray, which represented the level of expression of the corresponding genes in the tested cells, were detected and quantified using Scanner 3000 .

\section{Scanned images and data analysis}

The fluorescence intensities of the spots were extracted from the scanned microarray images, which consisted of high-density grid spots. The images were qualitatively and quantitatively analyzed and the signals were normalized using computers. Gene expression levels that differed by at least two-fold between samples were considered to be significantly different $(P<0.05)$. Under the given criteria, 1735 genes were identified as being differentially expressed in tumor sphere cells compared to the HGC-27 cells; among these, 610 genes were up-regulated and 1135 genes were down-regulated. The genes that were up-regulated or down-regulated more than 10-fold in the tumor sphere cells are listed in Tables 1 and 2, respectively. 
Table 1. Genes up-regulated more than 10-fold in tumor sphere cells.

\begin{tabular}{lll}
\hline Gene symbol & GenBank ID & Gene title \\
\hline TDO2 & NM_005651 & Tryptophan 2,3-dioxygenase \\
GDF15 & NM_004864 & Growth differentiation factor 15 \\
EGR1 & NM_001964 & Early growth response 1 \\
DUSP6 & NM_001946 & Dual specificity phosphatase 6 \\
ETV5 & NM_004454 & ETS variant 5 \\
PHLDB2 & NM_001134437 & Pleckstrin homology-like domain, family B, member 2 \\
MAFF & NM_001161572 & v-maf musculoaponeurotic fibrosarcoma oncogene homolog F (avian) \\
TRIB3 & NM_021158 & Tribbles homolog 3 (Drosophila) \\
COL1A2 & NM_000089 & Collagen, type I, alpha 2 \\
CREB5 & NM_001011666 & cAMP responsive element binding protein 5 \\
TOX & NM_014729 & Thymocyte selection-associated high mobility group box \\
EMP1 & NM_001423 & Epithelial membrane protein 1 \\
AKR1C3 & NM_003739 & Aldo-keto reductase family 1, member C3 (3-alpha hydroxysteroid dehydrogenase, t \\
ELK3 & NM_005230 & ELK3, ETS-domain protein (SRF accessory protein 2) \\
PROCR & NM_006404 & Protein C receptor, endothelial \\
LRRC3B & NM_052953 & Leucine rich repeat containing 3B \\
SHOX2 & NM_001163678 & Short stature homeobox 2 \\
\hline
\end{tabular}

Table 2. Genes down-regulated more than 10 -fold in tumor sphere cells.

\begin{tabular}{lll}
\hline Gene symbol & GenBank ID & Gene title \\
\hline TMEFF2 & NM_016192 & Transmembrane protein with EGF-like and two follistatin-like domains 2 \\
SFRP4 & NM_003014 & Secreted frizzled-related protein 4 \\
GRIK3 & NM_000831 & Glutamate receptor, ionotropic, kainate 3 \\
CHODL & NM_024944 & Chondrolectin \\
CNR1 & NM_001160226 & Cannabinoid receptor 1 (brain) \\
CNTN1 & NM_001843 & Contactin 1 \\
IGFBP5 & NM_000599 & Insulin-like growth factor binding protein 5 \\
EDNRA & NM_001166055 & Endothelin receptor type A \\
AMBN & NM_016519 & Ameloblastin (enamel matrix protein) \\
SEPP1 & NM_001085486 & Selenoprotein P, plasma, 1 \\
KBTBD10 & NM_006063 & Kelch repeat and BTB (POZ) domain containing 10 \\
DCT & NM_001129889 & Dopachrome tautomerase (dopachrome delta-isomerase, tyrosine-related protein 2) \\
\hline
\end{tabular}

The results of the gene chip analysis also revealed a close correlation between these differentially expressed genes and many aspects of cellular functioning, including cell signal transduction, cell growth, and tumorigenesis (Tables 3 and 4).

Table 3. Pathways with up-regulated genes in tumor sphere cells.

\begin{tabular}{l} 
Pathway title \\
\hline hsa05200: Pathways in cancer \\
hsa04010: MAPK signaling pathway \\
hsa04630: Jak-STAT signaling pathway \\
hsa00590: Arachidonic acid metabolism \\
hsa04512: ECM-receptor interaction \\
hsa05222: Small cell lung cancer \\
hsa04350: TGF-beta signaling pathway \\
hsa04660: T cell receptor signaling pathway \\
hsa04510: Focal adhesion \\
hsa04060: Cytokine-cytokine receptor interaction \\
\hline Some genes were up-regulated in the foll
\end{tabular}

Some genes were up-regulated in the following pathway of tumor sphere cells. 
Table 4. Pathways with down-regulated genes in tumor sphere cells.

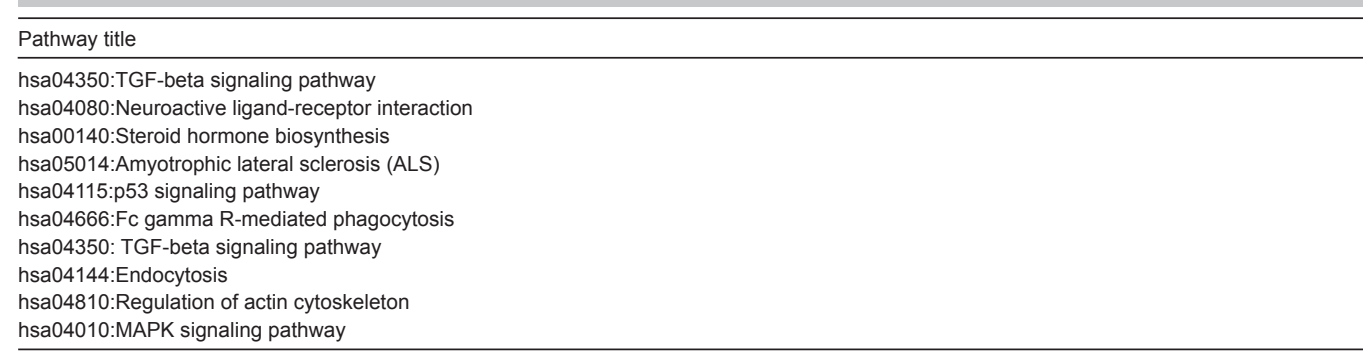

Some genes were down-regulated in the following pathway of tumor sphere cells.

\section{DISCUSSION}

According to the TSC theory, surviving tumor stem cells are the most likely cause of tumor recurrence, despite the bulk of tumor cells being destroyed by conventional therapy (Al-Hajj et al., 2003). Gene chip, a new technique developed in parallel with the Human Genome Project (HGP) (Nambiar et al., 2004), is currently widely used in gene sequencing, gene mutation detection, gene polymorphism analysis, and genetic testing of diseases. Gene chip analysis allows for the simultaneous detection of thousands of genes, as well as genome-wide gene profiling. This data could help elucidate gene functions, as well as interactions between the genes.

Our previous studies focused on exploring the growth characteristics of tumor sphere cells, and demonstrated their stem cell-like properties, such as reproducibility, clonogenicity, and high tumorigenicity (Shi et al., 2010, 2011). The aim of this study was to profile genes that are differentially expressed in tumor sphere cells when compared to HGC-27 cells. Through gene chip analysis, we identified 610 up-regulated and 1135 down-regulated genes, as described in the Results section. An in-depth analysis of genes whose expression levels differed by more than 10-fold between samples revealed the overexpression of some genes involved in tumorigenesis and metastasis, such as ETV5 and GDF15 (Wu et al., 2006; Breit et al., 2011). In addition, we also observed the down-regulation of some tumor suppressor genes, such as IGFBP5 and TMEFF2 (Costa et al., 2010; Jeter et al., 2011). These results supported the theory that tumor sphere cells might play a role in tumorigenesis. Additionally, genes that were rarely reported in previous studies, such as $P H L D B 2$ and $A K R 1 C 3$, were also identified in our study. The results of this study might therefore help elucidate the pathogenesis of gastric cancer on a molecular level, as well as identify specific molecular markers for diagnosis of, and potential targets for therapeutic drugs for gastric cancer.

\section{Conflicts of interest}

The authors declare no conflict of interest.

\section{REFERENCES}

Al-Hajj M, Wicha MS, Benito-Hernandez A, Morrison SJ, et al. (2003). Prospective identification of tumorigenic breast cancer cells. Proc. Natl. Acad. Sci. U.S.A. 100: 3983-3988.

Bertolini G, Roz L, Perego P, Tortoreto M, et al. (2009). Highly tumorigenic lung cancer CD133+ cells display stem-like features and are spared by cisplatin treatment. Proc. Natl. Acad. Sci. U.S.A. 106: 16281-16286.

Breit SN, Johnen H, Cook AD, Tsai VW, et al. (2011). The TGF-beta superfamily cytokine, MIC-1/GDF15: A pleotrophic cytokine 
with roles in inflammation, cancer and metabolism. Growth Factors 29: 187-195.

Costa VL, Henrique R, Danielsen SA, Duarte-Pereira S, et al. (2010). Three epigenetic biomarkers, GDF15, TMEFF2, and VIM, accurately predict bladder cancer from DNA-based analyses of urine samples. Clin. Cancer Res. 16: 5842-5851.

Eramo A, Lotti F, Sette G, Pilozzi E, et al. (2008). Identification and expansion of the tumorigenic lung cancer stem cell population. Cell Death Differ. 15: 504-514.

Jeter CR, Liu B, Liu X, Chen X, et al. (2011). NANOG promotes cancer stem cell characteristics and prostate cancer resistance to androgen deprivation. Oncogene 30: 3833-3845.

Levina V, Marrangoni A, Wang T, Parikh S, et al. (2010). Elimination of human lung cancer stem cells through targeting of the stem cell factor-c-kit autocrine signaling loop. Cancer Res. 70: 338-346.

Mimeault M, Hauke R, Mehta PP and Batra SK (2007). Recent advances in cancer stem/progenitor cell research: therapeutic implications for overcoming resistance to the most aggressive cancers. J. Cell Mol. Med. 11: 981-1011.

Nambiar S, Mirmohammadsadegh A, Bar A, Bardenheuer W, et al. (2004). Applications of array technology: melanoma research and diagnosis. Expert Rev. Mol. Diagn. 4: 549-558.

Reya T, Morrison SJ, Clarke MF and Weissman IL (2001). Stem cells, cancer, and cancer stem cells. Nature 414: 105-111.

Shi WH, Li C, Zhou S, Li L, et al. (2010). Human oral epithelial carcinoma of KB cells and SP cells in NOD/SCID mice subcutaneous tumor research. Modern Oncology 18: 2088-2090.

Shi WH, Liu JJ, Wang L, Sun W, et al. (2011). People ccl227 colon cancer cell tumor growth characteristics and nude mouse transplantation tumor formation research. Modern Oncology 19: 626-628.

Wu SH, Zhang J, Li Y and Li JM (2006). ETV5/MMP-7 in the expression of early cervical cancer tissue and its role in the transfer. Chin. J. Cancer 25: 315-319.

Yu SC, Ping YF, Yi L, Zhou ZH, et al. (2008). Isolation and characterization of cancer stem cells from a human glioblastoma cell line U87. Cancer Lett. 265: 124-134. 